\title{
Argument estimates of certain classes of P-Valent meromorphic functions involving certain operator
}

\author{
A. O. Mostafa ${ }^{1}$, M. K. Aouf ${ }^{2}$ and S. M. Madian ${ }^{3}$ \\ 1,2Department of Mathematics, Faculty of Science, \\ Mansoura University, Mansoura 35516, Egypt. \\ 3 Basic Sciences Department, Higher Institute of \\ Engineering and Technology, New Damietta, Egypt \\ 1adelaeg254@yahoo.com, ${ }^{2}$ mkaouf127@yahoo.com \\ 3samar_math@yahoo.com
}

\section{ABSTRACT}

In this paper, by making use of subordination, we investigate some inclusion relations and argument properties of certain classes of $p$-valent meromorphic functions involving certain operator.

\section{Indexing terms/Keywords}

Argument estimates, Hadamard product, certain operator, meromorphic functions.

\section{SUBJECT CLASSIFICATION}

2010 Mathematics Subject Classification: 30C45.

\section{Introduction}

For any integer $m>-p$, let $\sum_{p, m}$ denote the class of meromorphic functions $f(z)$ of the form:

$$
f(z)=z^{-p}+\sum_{k=m}^{\infty} a_{k} z^{k}(m>-p ; p \in \mathrm{N}=\{1,2, \cdots\})
$$

which are analytic and $p$-valent in the punctured open unit disc $\bigcup^{*}=\{z \in \mathrm{C}: 0<|z|<1\}=\bigcup \backslash\{0\}$. For convenience, we write $\sum_{p, 1-p}=\sum_{p}$ and $\sum_{1,0}=\sum$. If $f(z)$ and $g(z)$ are analytic in $U$, we say that $f(z)$ is subordinate to $g(z)$, written $f \prec g$ or $f(z) \prec g(z) \quad(z \in \mathrm{U})$, if there exists a Schwarz function $w(z)$ in $\bigcup$ with $w(0)=0$ and $|w(z)|<1 \quad(z \in \bigcup)$, such that $f(z)=g(w(z))(z \in \cup \cup$ Furthermore, if $g(z)$ is univalent in $U$, then the following equivalence relationship holds true (see [7] and [18]):

$$
f(z) \prec g(z) \Leftrightarrow f(0)=g(0) \text { and } f(\mathrm{U}) \subset g(\mathrm{U}) \text {. }
$$

For functions $f(z) \in \sum_{p, m}$, given by (1.1) and $g(z) \in \sum_{p, m}$ defined by

$$
g(z)=z^{-p}+\sum_{k=m}^{\infty} b_{k} z^{k} \quad(m>-p ; p \in \mathrm{N})
$$

the Hadamard product (or convolution) of $f(z)$ and $g(z)$ is given by 


$$
(f * g)(z)=z^{-p}+\sum_{k=m}^{\infty} a_{k} b_{k} z^{k}=(g * f)(z)
$$

For complex parameters $\alpha_{1}, \alpha_{2}, \ldots, \alpha_{q}$ and $\beta_{1}, \beta_{2}, \ldots, \beta_{s}\left(\alpha_{i}, \beta_{j} \notin \mathrm{Z}_{0}^{-}=\{0,-1,-2, \ldots\}, i=1,2, \ldots, q, j=1,2, \ldots, s\right)$, the generalized hypergeometric function ${ }_{q} F_{s}\left(\alpha_{1}, \alpha_{2}, \ldots, \alpha_{q} ; \beta_{1}, \beta_{2}, \ldots, \beta_{s} ; z\right)$ is defined by (see [22])

$$
\begin{gathered}
{ }_{q} F_{s}\left(\alpha_{1}, \alpha_{2}, \ldots, \alpha_{q} ; \beta_{1}, \beta_{2}, \ldots, \beta_{s} ; z\right)=\sum_{k=0}^{\infty} \frac{\left(\alpha_{1}\right)_{k} \ldots\left(\alpha_{q}\right)_{k}}{\left(\beta_{1}\right)_{k} \ldots\left(\beta_{s}\right)_{k}(1)_{k}} z^{k} \\
\left(q \leq s+1 ; s, q \in \mathrm{N}_{0}=\mathrm{N} \cup\{0\} ; z \in \mathrm{U}\right),
\end{gathered}
$$

where $(\theta)_{k}$, is the Pochhammer symbol defined in terms of the Gamma function $\Gamma$, by

$$
(\theta)_{v}=\frac{\Gamma(\theta+v)}{\Gamma(\theta)}= \begin{cases}1 & \left(v=0 ; \theta \in \mathrm{C}^{*}=\mathrm{C} \backslash\{0\}\right) \\ \theta(\theta+1) \ldots(\theta+v-1) & (v \in \mathrm{N} ; \theta \in \mathrm{C}) .\end{cases}
$$

Liu and Srivastava [16] and Aouf [4] investigated recently the operator $\Upsilon_{p, q . s}\left(\alpha_{1}, \alpha_{2}, \ldots, \alpha_{q}\right.$; $\left.\beta_{1}, \beta_{2}, \ldots, \beta_{s}\right): \sum_{p, m} \rightarrow \sum_{p, m}$, defined as follows:

$$
\begin{aligned}
\Upsilon_{p, q . s}\left(\alpha_{1}\right) & =\Upsilon_{p, q . s}\left(\alpha_{1}, \alpha_{2}, \ldots, \alpha_{q} ; \beta_{1}, \beta_{2}, \ldots, \beta_{s} ; z\right)=z^{-p} F_{s}\left(\alpha_{1}, \alpha_{2}, \ldots, \alpha_{q} ; \beta_{1}, \beta_{2}, \ldots, \beta_{s} ; z\right), \\
& =z^{-p}+\sum_{k=1-p}^{\infty} \frac{\left(\alpha_{1}\right)_{k+p} \ldots\left(\alpha_{q}\right)_{k+p}}{\left(\beta_{1}\right)_{k+p} \ldots\left(\beta_{s}\right)_{k+p}(1)_{k+p}} a_{k} z^{k} .
\end{aligned}
$$

With aid of the function $\Upsilon_{p, q . s}\left(\alpha_{1}\right)$ given by (1.2), consider the function $\Upsilon_{p, q . s}^{*}\left(\alpha_{1}\right)$ defined by:

$$
\Upsilon_{p, q . s}\left(\alpha_{1}\right) * \Upsilon_{p, q . s}^{*}\left(\alpha_{1}\right)=\frac{1}{z^{p}(1-z)^{\lambda+p}}\left(\lambda>-p ; p \in \mathrm{N} ; z \in \mathrm{U}^{*}\right)
$$

This function leads us to the following family of linear operators $\mathbf{M}_{p, q, s}^{\lambda, m}\left(\alpha_{1}\right): \sum_{p, m}^{\lambda} \rightarrow \sum_{p, m}^{\lambda}$, which are given by:

$$
\mathbf{M}_{p, q, s}^{\lambda, m}\left(\alpha_{1}\right)=\Upsilon_{p, q . s}^{*}\left(\alpha_{1}\right) * f(z)\left(f \in \sum_{p, m}\right) .
$$

The linear operator $\mathbf{M}_{p, q, s}^{\lambda, m}\left(\alpha_{1}\right)$ was defined by Patel and Patil [20] and Mostafa [17]. If $f(z)$ is given by (1.1), then from (1.4), we deduce that

$$
\mathbf{M}_{p, q, s}^{\lambda, m}\left(\alpha_{1}\right) f(z)=z^{-p}+\sum_{k=m}^{\infty} \frac{\left(\beta_{1}\right)_{k+p} \ldots\left(\beta_{s}\right)_{k+p}(\lambda+p)_{k+p}}{\left(\alpha_{1}\right)_{k+p} \ldots\left(\alpha_{q}\right)_{k+p}} a_{k} z^{k}
$$




$$
\left(f \in \sum_{p, m} ; \lambda, m>-p ; p \in \mathrm{N} ; z \in \mathrm{U}^{*}\right) .
$$

It is easily verified from (1.5) that (see [20] and [17])

$$
z\left(\mathbf{M}_{p, q, s}^{\lambda, m}\left(\alpha_{1}\right) f(z)\right)^{\prime}=(\lambda+p) \mathbf{M}_{p, q, s}^{\lambda+1, m}\left(\alpha_{1}\right) f(z)-(\lambda+2 p) \mathbf{M}_{p, q, s}^{\lambda, m}\left(\alpha_{1}\right) f(z)
$$

and

$$
z\left(\mathbf{M}_{p, q, s}^{\lambda, m}\left(\alpha_{1}+1\right) f(z)\right)^{\prime}=\alpha_{1} \mathbf{M}_{p, q, s}^{\lambda, m}\left(\alpha_{1}\right) f(z)-\left(\alpha_{1}+p\right) \mathbf{M}_{p, q, s}^{\lambda, m}\left(\alpha_{1}+1\right) f(z) .
$$

For a function $f \in \sum_{p}$ and $\mu>0$, let $F_{\mu, p}: \sum_{p} \rightarrow \sum_{p}$ be the integral operator defined by (see [13]):

$$
\begin{aligned}
& F_{\mu, p}(f)(z)=\frac{\mu}{z^{\mu+p}} \int_{0}^{z} t^{\mu+p-1} f(t) d t=z^{-p}+\sum_{k=1-p}^{\infty} \frac{\mu}{\mu+k+p} a_{k} z^{k}(18 \\
& \left(f \in \sum_{p} ; \mu>0 ; p \in \mathrm{N} ; z \in \mathrm{U}^{*}\right) .
\end{aligned}
$$

It follows from (1.8) that:

$$
z\left(\mathbf{M}_{p, q, s}^{\lambda, m}\left(\alpha_{1}\right) F_{\mu, p}(f)(z)\right)^{\prime}=\mu \mathbf{M}_{p, q, s}^{\lambda, m}\left(\alpha_{1}\right) f(z)-(\mu+p) \mathbf{M}_{p, q, s}^{\lambda, m}\left(\alpha_{1}\right) F_{\mu, p}(f)(z) .
$$

We note that:

Putting $\lambda=1-p(p \in \mathrm{N})$ in (1.5), then the operator $\mathbf{M}_{p, q, s}^{1-p, m}\left(\alpha_{1}\right)$ reduces to the operator $\mathbf{M}_{p, q, s}^{m}\left(\alpha_{1}\right)$, defined by:

$$
\mathbf{M}_{p, q, s}^{m}\left(\alpha_{1}\right) f(z)=z^{-p}+\sum_{k=m}^{\infty} \frac{\left(\beta_{1}\right)_{k+p} \ldots\left(\beta_{s}\right)_{k+p}}{\left(\alpha_{1}\right)_{k+p} \ldots\left(\alpha_{q}\right)_{k+p}} a_{k} z^{k} \quad\left(f \in \sum_{p, m} ; m>-p ; p \in \mathrm{N} ; z \in \mathbf{U}^{*}\right)
$$

Also, by specializing the parameters $\lambda, m, p, \alpha_{i}(i=1,2, \ldots, q), \beta_{j}(j=1,2, \ldots, s), q$ and $s$, we have:

(i) $\mathbf{M}_{p, 2,1}^{0, m}(p, p ; p) f(z)=\mathbf{M}_{p, 2,1}^{1}(p+1, p ; p) f(z)=f(z)(p \in \mathrm{N})$;

(ii) $\mathbf{M}_{p, 2,1}^{1, m}(p, p ; p) f(z)=\frac{2 p f(z)+z f(z)}{p}(p \in \mathrm{N})$;

(iii) $\mathbf{M}_{p, 2,1}^{2, m}(p+1, p ; p) f(z)=\frac{(2 p+1) f(z)+z f^{\prime}(z)}{p+1}(p \in \mathrm{N})$;

(iv) $\mathbf{M}_{p, 2,1}^{n, 0}(a, 1 ; a) f(z)=D^{n+p-1} f(z)(n>-p, a>0, p \in \mathrm{N})$ (see Yang [23] and Aouf ( [2] and [3])), which for $p=1$ reduces to the operator $D^{n} f(z) \quad(n>-1) \quad$ ( see Cho [8] );

(v) $\mathbf{M}_{p, 2,1}^{0, m}(p+1, p ; p) f(z)=\frac{p}{z^{2 p}} \int_{0}^{z} t^{2 p-1} f(t) d t(p \in \mathrm{N})$;

(vi) $\mathbf{M}_{p, 2,1}^{1-p, m}(\mu+1,1 ; \mu) f(z)=F_{\mu, p}(f)(z) \quad(p \in \mathrm{N}, \mu>0)$, this integral operator is defined by (1.8);

(vii) $\mathbf{M}_{p, 2,1}^{\lambda, m}(c, p+\lambda ; a) f(z)=L_{p}(a ; c) f(z)\left(p \in \mathrm{N}, a \in \mathrm{R}, c \in \mathrm{R} \backslash \mathrm{Z}_{0}^{-}\right)$( see Liu [15]); 
(viii) $\mathbf{M}_{p, 2,1}^{\lambda, 1-p}(a, 1 ; c) f(z)=L_{p}^{\lambda}(a ; c) f(z)\left(\lambda>-p, p \in \mathrm{N}, a, c \in \mathrm{R} \backslash \mathrm{Z}_{0}^{-}\right)$( see Aouf et al. [5] ), which for $p=1$ reduces to $L^{\lambda}(a ; c)$ ( see Aghalary [1]);

(ix) $\mathbf{M}_{p, 2,1}^{\eta-p, 1-p}(n+p, \eta ; \eta) f(z)=I_{n+p-1, \eta} f(z)(\eta>0, n>-p, p \in \mathrm{N}) \quad$ (see Aouf and Xu [6]), which $p=1$ reduces to $I_{n, \eta}$ ( see Yuan et al. [24]);

(x) $\mathbf{M}_{1, q, s}^{\sigma-1,0}\left(\alpha_{1}\right) f(z)=H_{\sigma, q, s}\left(\alpha_{1}\right) f(z)(\sigma>0)$ ( see Cho and Kim [9]);

(xi) $\mathbf{M}_{1,2,1}^{x-1,0}(a, 1 ; c) f(z)=I_{x}(a, c) f(z) \quad\left(x>0, a, c \in \mathrm{R} \backslash \mathrm{Z}_{0}^{-}\right) \quad$ (Cho and Noor [10]).

Let $M$ be the class of functions $h(z)$ which are analytic and univalent in $U$ and for which $h(\mathrm{U})$ is convex with $h(0)=1$ and $\operatorname{Re}\{h(z)\}>0, z \in U$.

Now, by using the linear operator $\mathbf{M}_{p, q, s}^{\lambda, m}\left(\alpha_{1}\right)$, we define a subclass of $\sum_{p, m}$ by

$$
\sum_{p, q, s}^{\lambda, m}\left(\alpha_{1} ; h\right)=\left\{f: f \in \sum_{p, m} \text { and }-\frac{z\left(\mathbf{M}_{p, q, s}^{\lambda, m}\left(\alpha_{1}\right) f(z)\right)^{\prime}}{p \mathbf{M}_{p, q, s}^{\lambda, m}\left(\alpha_{1}\right) f(z)} \prec h(z)(h \in M ; z \in \mathrm{U})\right\}(1 .
$$

We also set

$$
\sum_{p, q, s}^{\lambda, m}\left(\alpha_{1} ; \frac{1+A z}{1+B z}\right)=\sum_{p, q, s}^{\lambda, m}\left(\alpha_{1} ; A, B\right) \quad(-1<B<A \leq 1 ; z \in \mathrm{U}) .
$$

From (1.10) and (1.11) and by using the result of Silverman and Silvia [21], we observe that a function $f(z)$ is in the class $\sum_{p, q, s}^{\lambda, m}\left(\alpha_{1} ; A, B\right)$ if and only if

$$
\left|\frac{z\left(\mathbf{M}_{p, q, s}^{\lambda, m}\left(\alpha_{1}\right) f(z)\right)^{\prime}}{\mathbf{M}_{p, q, s}^{\lambda, m}\left(\alpha_{1}\right) f(z)}+\frac{p(1-A B)}{1-B^{2}}\right|<\frac{p(A-B)}{1-B^{2}}(-1<B<A \leq 1 ; z \in \mathrm{U}) .
$$

In the present paper, we investigate some inclusion relationships and argument properties of certain meromorphically $p$-valent functions in $\mathrm{U}^{*}$ in connection with the linear operator $\mathbf{M}_{p, q, s}^{\lambda, m}\left(\alpha_{1}\right)$.

\section{Preliminaries}

In order to prove our main results, we need to the following lemmas.

Lemma 1 [12]. Let $\beta$ and $v$ be complex constants and let $h(z)$ be convex (univalent) in $U$ with $h(0)=1$ and $\operatorname{Re}\{\beta h(z)+v\}>0$. If $q(z)=1+q_{1} z+\ldots$ is analytic in $\mathrm{U}$, then

$$
q(z)+\frac{z q^{\prime}(z)}{\beta q(z)+v} \prec h(z),
$$


implies

$$
q(z) \prec h(z) .
$$

Lemma 2 [18]. Let $h(z)$ be convex (univalent) in $U$ and $\psi(z)$ be analytic in $U$ with $\operatorname{Re}\{\psi(z)\} \geq 0$. If $q$ is analytic in $\mathrm{U}$ and $q(0)=h(0)$, then

$$
q(z)+\psi(z) z q^{\prime}(z) \prec h(z),
$$

implies

$$
q(z) \prec h(z) .
$$

Lemma 3 [19]. Let $q(z)$ be analytic in $U$, with $q(0)=1$ and $q(z) \neq 0 \quad(z \in \mathrm{U})$. If there exists a point $z_{0}$ 圆 U, such that

$$
|\arg q(z)|<\frac{\pi}{2} \tau \text { for }|z|<\left|z_{0}\right|
$$

and

$$
\left|\arg q\left(z_{0}\right)\right|=\frac{\pi}{2} \tau \quad(0<\tau \leq 1) .
$$

Then we have

$$
\frac{z_{0} q^{\prime}\left(z_{0}\right)}{q\left(z_{0}\right)}=i x \tau,
$$

where

$$
\begin{aligned}
& x \geq \frac{1}{2}\left(b+\frac{1}{b}\right) \text { when } \arg q\left(z_{0}\right)=\frac{\pi}{2} \tau, \\
& x \geq-\frac{1}{2}\left(b+\frac{1}{b}\right) \text { when } \arg q\left(z_{0}\right)=-\frac{\pi}{2} \tau
\end{aligned}
$$

and

$$
q\left(z_{0}\right)^{\frac{1}{\tau}}= \pm i b \quad(b>0) .
$$

\section{Some inclusion relationships}

By using Lemma 1, we obtain the following results: 
Theorem 1. Let $h(z) \in M$ with $\max _{z \in \mathrm{U}} \operatorname{Re}\{h(z)\}<\min \left\{\frac{\lambda+2 p}{p}, \frac{\alpha_{1}+p}{p}\right\} \quad(\{\stackrel{\leftrightarrow}{*}, \quad p \in \mathrm{N})$. Then

$$
\sum_{p, q, s}^{\lambda+1, m}\left(\alpha_{1} ; h\right) \subset \sum_{p, q, s}^{\lambda, m}\left(\alpha_{1} ; h\right) \subset \sum_{p, q, s}^{\lambda, m}\left(\alpha_{1}+1 ; h\right) .
$$

Proof. To prove the first part, we show that $\sum_{p, q, s}^{\lambda+1, m}\left(\alpha_{1} ; h\right) \subset \sum_{p, q, s}^{\lambda, m}\left(\alpha_{1} ; h\right)$. Let $f \in \sum_{p, q, s}^{\lambda+1, m}\left(\alpha_{1} ; h\right)$ and set

$$
R(z)=-\frac{z\left(\mathbf{M}_{p, q, s}^{\lambda, m}\left(\alpha_{1}\right) f(z)\right)^{\prime}}{p \mathbf{M}_{p, q, s}^{\lambda, m}\left(\alpha_{1}\right) f(z)}(z \in \mathrm{U}),
$$

where $R(z)$ is analytic with $R(0)=1$. Using (1.6) in (3.1), we obtain

$$
p R(z)-(\lambda+2 p)=-(\lambda+p) \frac{\mathbf{M}_{p, q, s}^{\lambda+1, m}\left(\alpha_{1}\right) f(z)}{\mathbf{M}_{p, q, s}^{\lambda, m}\left(\alpha_{1}\right) f(z)} .
$$

Differentiating (3.2) logarithmically with respect to $z$ and multiplying by $z$, we have

$$
R(z)+\frac{z R^{\prime}(z)}{-p R(z)+\lambda+2 p}=-\frac{z\left(\mathbf{M}_{p, q, s}^{\lambda+1, m}\left(\alpha_{1}\right) f(z)\right)^{\prime}}{p \mathbf{M}_{p, q, s}^{\lambda+1, m}\left(\alpha_{1}\right) f(z)} \prec h(z),
$$

from Lemma 1, it follows that $R(z) \prec h(z)$ in $\mathrm{U}$, that is, that $f \in \sum_{p, q, s}^{\lambda, m}\left(\alpha_{1} ; h\right)$.

To prove the second part, let $f \in \sum_{p, q, s}^{\lambda, m}\left(\alpha_{1} ; h\right)$ and put

$$
s(z)=-\frac{z\left(\mathbf{M}_{p, q, s}^{\lambda, m}\left(\alpha_{1}+1\right) f(z)\right)^{\prime}}{p \mathbf{M}_{p, q, s}^{\lambda, m}\left(\alpha_{1}+1\right) f(z)}(z \in \mathrm{U}),
$$

then, by using the arguments similar to those detailed above and using (1.7) instead of (1.6), it follows that $s(z) \prec h(z)$ in $\mathrm{U}$, which implies $f \in \sum_{p, q, s}^{\lambda, m}\left(\alpha_{1}+1 ; h\right)$. Therefore we compelet the proof of Theorem 1.

Taking $h(z)=\frac{1+A z}{1+B z} \quad(-1<B<A \leq 1)$ in Theorem 1, we have

Corollary 1. Let $\frac{1+A}{1+B} \quad<\min \left\{\frac{\lambda+2 p}{p}, \frac{\alpha_{1}+p}{p}\right\}$ and $-1<B<A \leq 1$. Then

$$
\sum_{p, q, s}^{\lambda+1, m}\left(\alpha_{1} ; A, B\right) \subset \sum_{p, q, s}^{\lambda, m}\left(\alpha_{1} ; A, B\right) \subset \sum_{p, q, s}^{\lambda, m}\left(\alpha_{1}+1 ; A, B\right) .
$$

Theorem 2. Let $h(z) \in M$ with $\operatorname{Re}\{h(z)\}<\frac{\mu+p}{p}(\mu>0)$, if $f \in \sum_{p, q, s}^{\lambda, m}\left(\alpha_{1} ; h\right)$, then $F_{\mu, p}(f) \in \sum_{p, q, s}^{\lambda, m}\left(\alpha_{1} ; h\right)$, where $F_{\mu, p}(f)$ is defined by (1.8) .

Proof. Let $f \in \sum_{p, q, s}^{\lambda, m}\left(\alpha_{1} ; h\right)$ and set

$$
L(z)=-\frac{z\left(\mathbf{M}_{p, q, s}^{\lambda, m}\left(\alpha_{1}\right) F_{\mu, p}(f)(z)\right)^{\prime}}{p \mathbf{M}_{p, q, s}^{\lambda, m}\left(\alpha_{1}\right) F_{\mu, p}(f)(z)}(z \in U),
$$

where $L(z)$ is analytic with $L(0)=1$. Applying (1.9) to (3.4), we get 


$$
p L(z)-(\mu+p)=-\mu \frac{\mathbf{M}_{p, q, s}^{\lambda, m}\left(\alpha_{1}\right) f(z)}{\mathbf{M}_{p, q, s}^{\lambda, m}\left(\alpha_{1}\right) F_{\mu, p}(f)(z)} .
$$

Differentiating (3.5) logarithmically with respect to $z$ and multiplying by $z$, we have

$$
L(z)+\frac{z L^{\prime}(z)}{-p L(z)+\mu+p}=-\frac{z\left(\mathbf{M}_{p, q, s}^{\lambda, m}\left(\alpha_{1}\right) f(z)\right)^{\prime}}{p \mathbf{M}_{p, q, s}^{\lambda, m}\left(\alpha_{1}\right) f(z)} \prec h(z) .
$$

Hence, by virtue of Lemma 1 , we conclude that $L(z) \prec h(z)$ in $\mathrm{U}$, which implies $F_{\mu, p}(f) \in \sum_{p, q, s}^{\lambda, m}\left(\alpha_{1} ; h\right)$. This compelets the proof of Theorem 2.

Taking $h(z)=\frac{1+A z}{1+B z} \quad(-1<B<A \leq 1)$ in Theorem 2, we have

Corollary 2. Let $\frac{1+A}{1+B} \quad<\frac{\mu+p}{p}(\mu>0)$ and $-1<B<A \leq 1$, if $f \in \sum_{p, q, s}^{\lambda, m}\left(\alpha_{1} ; A, B\right)$, then $F_{\mu, p}(f) \in \sum_{p, q, s}^{\lambda, m}\left(\alpha_{1} ; A, B\right)$.

\section{Some argument properties}

Theorem 3. Let $f(z) \in \sum_{p, m}, \quad 0<\delta \leq 1, \quad 0 \leq \ell<p$ and

$$
\lambda \geq \frac{p(A-B)}{1+B}-p(-1<B<A \leq 1 ; p \in \mathrm{N}) .
$$

If

$$
\left|\arg \left(-\frac{z\left(\mathbf{M}_{p, q, s}^{\lambda+1, m}\left(\alpha_{1}\right) f(z)\right)^{\prime}}{\mathbf{M}_{p, q, s}^{\lambda+1, m}\left(\alpha_{1}\right) g(z)}-\ell\right)\right|<\frac{\pi}{2} \delta,
$$

for some $g(z) \in \sum_{p, q, s}^{\lambda+1, m}\left(\alpha_{1} ; A, B\right)$, then

$$
\left|\arg \left(-\frac{z\left(\mathbf{M}_{p, q, s}^{\lambda, m}\left(\alpha_{1}\right) f(z)\right)^{\prime}}{\mathbf{M}_{p, q, s}^{\lambda, m}\left(\alpha_{1}\right) g(z)}-\ell\right)\right|<\frac{\pi}{2} \tau,
$$

where $\tau(0<\tau \leq 1)$ is the solution of the equation

$$
\delta=\tau+\frac{2}{\pi} \tan ^{-1}\left(\frac{\tau \cos \frac{\pi}{2} t(A, B)}{\frac{(\lambda+p)(1-B)+p(A-B)}{1-B}+\tau \sin \frac{\pi}{2} t(A, B)}\right)
$$

and

$$
t(A, B)=\frac{2}{\pi} \sin ^{-1}\left(\frac{p(A-B)}{(\lambda+2 p)\left(1-B^{2}\right)-p(1-A B)}\right)
$$

Proof. Let 


$$
q(z)=\frac{1}{p-\ell}\left(-\frac{z\left(\mathbf{M}_{p, q, s}^{\lambda, m}\left(\alpha_{1}\right) f(z)\right)^{\prime}}{\mathbf{M}_{p, q, s}^{\lambda, m}\left(\alpha_{1}\right) g(z)}-\ell\right),
$$

where $q(z)$ is analytic with $q(0)=1$. Applying the identity (1.6), we have

$$
[-(p-\ell) q(z)-\ell] \mathbf{M}_{p, q, s}^{\lambda, m}\left(\alpha_{1}\right) g(z)=(\lambda+p) \mathbf{M}_{p, q, s}^{\lambda+1, m}\left(\alpha_{1}\right) f(z)-(\lambda+2 p) \mathbf{M}_{p, q, s}^{\lambda, m}\left(\alpha_{1}\right) f(z) .
$$

Differentiating (4.4) with respect to $z$ and multiplying by $z$, we obtain

$$
\begin{gathered}
-(p-\ell) z q^{\prime}(z) \mathbf{M}_{p, q, s}^{\lambda, m}\left(\alpha_{1}\right) g(z)+[-(p-\ell) q(z)-\ell] z\left(\mathbf{M}_{p, q, s}^{\lambda, m}\left(\alpha_{1}\right) g(z)\right)^{\prime} \\
=(\lambda+p) z\left(\mathbf{M}_{p, q, s}^{\lambda+1, m}\left(\alpha_{1}\right) f(z)\right)^{\prime}-(\lambda+2 p) z\left(\mathbf{M}_{p, q, s}^{\lambda, m}\left(\alpha_{1}\right) f(z)\right)^{\prime} .
\end{gathered}
$$

Then, by using (4.3), (4.4) and (4.5), we have

$$
\frac{1}{p-\ell}\left(-\frac{z\left(\mathbf{M}_{p, q, s}^{\lambda+1, m}\left(\alpha_{1}\right) f(z)\right)^{\prime}}{\mathbf{M}_{p, q, s}^{\lambda+1, m}\left(\alpha_{1}\right) g(z)}-\ell\right)=q(z)+\frac{z q^{\prime}(z)}{-r(z)+\lambda+2 p}
$$

where

$$
r(z)=-\frac{z\left(\mathbf{M}_{p, q, s}^{\lambda, m}\left(\alpha_{1}\right) g(z)\right)^{\prime}}{\mathbf{M}_{p, q, s}^{\lambda, m}\left(\alpha_{1}\right) g(z)} .
$$

From Corollary 1 , since $g(z) \in \sum_{p, q, s}^{\lambda+1, m}\left(\alpha_{1} ; A, B\right)$, then $g(z) \in \sum_{p, q, s}^{\lambda, m}\left(\alpha_{1} ; A, B\right)$, which from (1.12) leads to

$$
r(z) \prec p \frac{1+A z}{1+B z} .
$$

Letting

$$
-r(z)+\lambda+2 p=\rho e^{i \frac{\pi}{2} \phi}(z \in \mathrm{U})
$$

then from (1.12) we have

$$
\frac{(\lambda+p)(1+B)-p(A-B)}{1+B}<\rho<\frac{(\lambda+p)(1-B)+p(A-B)}{1-B}
$$

and

$$
-t(A, B)<\phi<t(A, B)
$$

where $t$ is defined by (4.2). 
Let $h$ be a function which maps $U$ onto the angular domain $\left\{\omega:|\arg \omega|<\frac{\pi}{2} \delta\right\}$ with $h(0)=1$. Applying Lemma 2, for this $h$ with $\psi(z)=\frac{1}{-r(z)+\lambda+2 p}$, we see that $\operatorname{Re}\{q(z)\}>0$ in $U$ and hence $q(z) \neq 0$ in $\mathrm{U}$. If there exists a point $z_{0} \in \mathrm{U}$ such that the conditions (2.1) and (2.2) are satisfied, then by Lemma 3, we have (2.3) under the restrictions (2.4) and (2.5).

At first, suppose that $q\left(z_{0}\right)^{\frac{1}{\tau}}=i b \quad(b>0)$. Then

$$
\begin{aligned}
& \arg \left[-\frac{1}{p-\ell}\left(\frac{z_{0}\left(\mathbf{M}_{p, q, s}^{\lambda+1, m}\left(\alpha_{1}\right) f\left(z_{0}\right)\right)^{\prime}}{\mathbf{M}_{p, q, s}^{\lambda+1, m}\left(\alpha_{1}\right) g\left(z_{0}\right)}+\ell\right)\right] \\
= & \arg \left(q\left(z_{0}\right)+\frac{z_{0} q^{\prime}\left(z_{0}\right)}{-r\left(z_{0}\right)+\lambda+2 p}\right)=\frac{\pi}{2} \tau+\arg \left(1+i \ell \tau\left(\rho e^{i \frac{\pi}{2} \phi}\right)^{-1}\right) \\
= & \frac{\pi}{2} \tau+\tan ^{-1}\left(\frac{\ell \tau \sin \frac{\pi}{2}(1-\phi)}{\rho+\ell \tau \cos \frac{\pi}{2}(1-\phi)}\right) \\
\geq & \frac{\pi}{2} \tau+\tan ^{-1}\left(\frac{\tau \cos \frac{\pi}{2} t(A, B)}{\frac{(\lambda+p)(1-B)+p(A-B)}{1-B}+\tau \sin \frac{\pi}{2} t(A, B)}\right)=\frac{\pi}{2} \delta,
\end{aligned}
$$

where $\Varangle$ and $t(A, B)$ are given by (4.1) and (4.2), respectively. This contradicts to the assumption of the theorem.

Next, suppose that $q\left(z_{0}\right)^{\frac{1}{\alpha}}=-i b \quad(b>0)$. Applying the same method as the above, we have

$$
\begin{aligned}
& \arg \left[-\frac{1}{p-\ell}\left(\frac{z_{0}\left(\mathbf{M}_{p, q, s}^{\lambda+1, m}\left(\alpha_{1}\right) f\left(z_{0}\right)\right)^{\prime}}{\mathbf{M}_{p, q, s}^{\lambda+1, m}\left(\alpha_{1}\right) g\left(z_{0}\right)}+\ell\right)\right] \\
\leq & -\frac{\pi}{2} \tau-\tan ^{-1}\left(\frac{\tau \cos \frac{\pi}{2} t(A, B)}{\frac{(\lambda+p)(1-B)+p(A-B)}{1-B}+\tau \sin \frac{\pi}{2} t(A, B)}\right)=-\frac{\pi}{2} \delta,
\end{aligned}
$$

where $\Varangle$ and $t(A, B)$ are given by (4.1) and (4.2), respectively, which contradicts the assumption. This completes the proof of Theorem 3.

Taking $q=2, s=\lambda=\delta=A=1, B=0$ and $\alpha_{1}=\alpha_{2}=\beta_{1}=p(p \in \mathrm{N})$ in Theorem 3, we have the following corollary:

Corollary 3. Let $f(z) \in \sum_{p, m}$. If

$$
-\operatorname{Re}\left\{\frac{z\left(2(p+1)(2 p+1) f^{\prime}(z)+4(1+p) z f^{\prime \prime}(z)+z^{2} f^{\prime \prime \prime}(z)\right)}{2 p(2 p+1) g(z)+2(2 p+1) z g^{\prime}(z)+z^{2} g^{\prime \prime}(z)}\right\}>\ell(0 \leq \ell<p),
$$

for some $g(z) \in \sum_{p, m}$ satisfying the condition 


$$
\left|\frac{z\left(2(p+1)(2 p+1) g^{\prime}(z)+4(p+1) z g^{\prime \prime}(z)+z^{2} g^{\prime \prime \prime}(z)\right)}{2 p(2 p+1) g(z)+2(2 p+1) z g^{\prime}(z)+z^{2} g^{\prime \prime}(z)}+p\right|<p,
$$

then

$$
-\operatorname{Re}\left\{\frac{z\left((2 p+1) f^{\prime}(z)+z f^{\prime \prime}(z)\right)}{z f^{\prime}(z)+2 p f(z)}\right\}>\ell
$$

Taking $q=2, s=\delta=A=1, \lambda=2, B=0, \alpha_{1}=p+1$ and $\alpha_{2}=\beta_{1}=p \quad(p \in \mathrm{N})$ in Theorem 3, we have the following corollary:

Corollary 4. Let $f(z) \in \sum_{p, m}$. If

$$
-\operatorname{Re}\left\{\frac{z\left(2((p+1))(2 p+3) f^{\prime}(z)+2(2 p+3) z f^{\prime \prime}(z)+z^{2} f^{\prime \prime \prime}(z)\right)}{2(p+1)(2 p+1) g(z)+4(p+1) z g^{\prime}(z)+z^{2} g^{\prime \prime}(z)}\right\}>\ell \quad(0 \leq \ell<p),
$$

for some $g(z) \in \sum_{p, m}$ satisfying the condition

$$
\left|\frac{z\left(2((p+1))(2 p+3) g^{\prime}(z)+2(2 p+3) z g^{\prime \prime}(z)+z^{2} g^{\prime \prime \prime}(z)\right)}{2(p+1)(2 p+1) g(z)+4(p+1) z g^{\prime}(z)+z^{2} g^{\prime \prime}(z)}+p\right|<p,
$$

then

$$
-\operatorname{Re}\left\{\frac{z\left(2(p+1) f^{\prime}(z)+z f^{\prime \prime}(z)\right)}{(2 p+1) g(z)+z g^{\prime}(z)}\right\}>\ell
$$

Taking $q=2, s=1, \lambda=\eta-p, \quad m=1-p, \alpha_{1}=n+p \quad$ and $\quad \alpha_{2}=\beta_{1}=\eta \quad(\eta>0, n>-p, p \in \mathrm{N}) \quad$ in Theorem 3 , we have the following corollary:

Corollary 5. Let $f(z) \in \sum_{p}, \quad 0<\delta \leq 1, \quad 0 \leq \ell<p$ and

$$
\eta \geq \frac{p(A-B)}{1+B} \quad(-1<B<A \leq 1 ; p \in \mathrm{N}) .
$$

If

$$
\left|\arg \left(-\frac{z\left(I_{n+p-1, \eta+1} f(z)\right)^{\prime}}{I_{n+p-1, \eta+1} g(z)}-\ell\right)\right|<\frac{\pi}{2} \delta,
$$

for some $g(z) \in \sum_{p, m}$ satisfying the conditon

$$
-\frac{z\left(I_{n+p-1, \eta} g(z)\right)^{\prime}}{I_{n+p-1, \eta} g(z)} \prec p \frac{1+A z}{1+B z},
$$

then 


$$
\left|\arg \left(-\frac{z\left(I_{n+p-1, \eta} f(z)\right)^{\prime}}{I_{n+p-1, \eta} g(z)}-\ell\right)\right|<\frac{\pi}{2} \tau,
$$

where $\tau(0<\tau \leq 1)$ is the solution of the equation (4.1) with $\lambda=\eta-p(\eta>0)$.

Taking $p=1$ in Corollary 5, we have the following corollary:

Corollary 6. Let $f(z) \in \sum, 0<\delta \leq 1,0 \leq \ell<1$ and

$$
\eta \geq \frac{A-B}{1+B}(-1<B<A \leq 1)
$$

If

$$
\left|\arg \left(-\frac{z\left(I_{n, \eta+1} f(z)\right)^{\prime}}{I_{n, \eta+1} g(z)}-\ell\right)\right|<\frac{\pi}{2} \delta,
$$

for some $g(z) \in \sum$ satisfying the conditon

$$
-\frac{z\left(I_{n, \eta} g(z)\right)^{\prime}}{I_{n, \eta} g(z)} \prec \frac{1+A z}{1+B z},
$$

then

$$
\left|\arg \left(-\frac{z\left(I_{n, \eta} f(z)\right)^{\prime}}{I_{n, \eta} g(z)}-\ell\right)\right|<\frac{\pi}{2} \tau,
$$

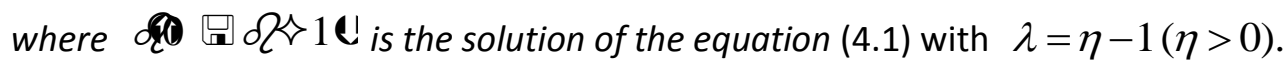

The proof of the next theorem is akin to that of Theorem 3 and so, we omit it.

Theorem 4. Let $f(z) \in \sum_{p, m}, \quad 0<\delta \leq 1, \ell>p$ and

$$
\lambda \geq \frac{p(A-B)}{1+B}-p \quad(-1<B<A \leq 1 ; p \in \mathrm{N}) .
$$

If

$$
\left|\arg \left(\frac{z\left(\mathbf{M}_{p, q, s}^{\lambda+1, m}\left(\alpha_{1}\right) f(z)\right)^{\prime}}{\mathbf{M}_{p, q, s}^{\lambda+1, m}\left(\alpha_{1}\right) g(z)}+\ell\right)\right|<\frac{\pi}{2} \delta
$$

for some $g(z) \in \sum_{p, q, s}^{\lambda+1, m}\left(\alpha_{1} ; A, B\right)$, then

$$
\left|\arg \left(\frac{z\left(\mathbf{M}_{p, q, s}^{\lambda, m}\left(\alpha_{1}\right) f(z)\right)^{\prime}}{\mathbf{M}_{p, q, s}^{\lambda, m}\left(\alpha_{1}\right) g(z)}+\ell\right)\right|<\frac{\pi}{2} \tau,
$$


where $\tau(0<\tau \leq 1)$ is the solution of equation (4.1).

Theorem 5. Let $f(z) \in \sum_{p, m}, \quad 0<\delta \leq 1,0 \leq \ell<p$ and

$$
\alpha_{1} \geq \frac{p(A-B)}{1+B} \quad(-1<B<A \leq 1 ; p \in \mathrm{N})
$$

If

$$
\left|\arg \left(-\frac{z\left(\mathbf{M}_{p, q, s}^{\lambda, m}\left(\alpha_{1}\right) f(z)\right)^{\prime}}{\mathbf{M}_{p, q, s}^{\lambda, m}\left(\alpha_{1}\right) g(z)}-\ell\right)\right|<\frac{\pi}{2} \delta
$$

for some $g(z) \in \sum_{p, q, s}^{\lambda, m}\left(\alpha_{1} ; A, B\right)$, then

$$
\left|\arg \left(-\frac{z\left(\mathbf{M}_{p, q, s}^{\lambda, m}\left(\alpha_{1}+1\right) f(z)\right)^{\prime}}{\mathbf{M}_{p, q, s}^{\lambda, m}\left(\alpha_{1}+1\right) g(z)}-\ell\right)\right|<\frac{\pi}{2} \tau,
$$

where $\tau(0<\tau \leq 1)$ is the solution of equation (4.1).

Proof. Let

$$
X(z)=\frac{1}{p-\ell}\left(-\frac{z\left(\mathbf{M}_{p, q, s}^{\lambda, m}\left(\alpha_{1}+1\right) f(z)\right)^{\prime}}{\mathbf{M}_{p, q, s}^{\lambda, m}\left(\alpha_{1}+1\right) g(z)}-\ell\right)
$$

where $X(z)$ is analytic with $X(0)=1$. Using (1.7), we have

$$
[-(p-\ell) X(z)-\ell] \mathbf{M}_{p, q, s}^{\lambda, m}\left(\alpha_{1}+1\right) g(z)=\alpha_{1} \mathbf{M}_{p, q, s}^{\lambda, m}\left(\alpha_{1}\right) f(z)-\left(\alpha_{1}+p\right) \mathbf{M}_{p, q, s}^{\lambda, m}\left(\alpha_{1}\right) f(z) .
$$

Differentiating (4.9) with respect to $z$ and multiplying by $z$, we obtain

$$
\begin{gathered}
-(p-\ell) z X^{\prime}(z) \mathbf{M}_{p, q, s}^{\lambda, m}\left(\alpha_{1}+1\right) g(z)+[-(p-\ell) X(z)-\ell] z\left(\mathbf{M}_{p, q, s}^{\lambda, m}\left(\alpha_{1}+1\right) g(z)\right)^{\prime} \\
=\alpha_{1} z\left(\mathbf{M}_{p, q, s}^{\lambda, m}\left(\alpha_{1}\right) f(z)\right)^{\prime}-\left(\alpha_{1}+p\right) z\left(\mathbf{M}_{p, q, s}^{\lambda, m}\left(\alpha_{1}\right) f(z)\right)^{\prime}
\end{gathered}
$$

Then, by using (4.8), (4.9) and (4.10), we have

$$
\frac{1}{p-?}\left(-\frac{z\left(\mathbf{M}_{p, q, s}^{\lambda, m}\left(\alpha_{1}\right) f(z)\right)^{\prime}}{\mathbf{M}_{p, q, s}^{\lambda, m}\left(\alpha_{1}\right) g(z)}-\ell\right)=X(z)+\frac{z X^{\prime}(z)}{-j(z)+\alpha_{1}+p}
$$

where

$$
j(z)=-\frac{z\left(\mathbf{M}_{p, q, s}^{\lambda, m}\left(\alpha_{1}+1\right) g(z)\right)^{\prime}}{\mathbf{M}_{p, q, s}^{\lambda, m}\left(\alpha_{1}+1\right) g(z)}
$$


The remaining part of the proof is similar to that of Theorem 3 and so we omit it.

Taking $q=2, s=1, \lambda=\eta-p, \quad m=1-p, \alpha_{1}=n+p \quad$ and $\quad \alpha_{2}=\beta_{1}=\eta \quad(\eta>0, n>-p, p \in \mathrm{N}) \quad$ in Theorem 5, we have the following corollary:

Corollary 7. Let $f(z) \in \sum_{p, m}, \quad 0<\delta \leq 1,0 \leq \ell<p$ and

$$
n \geq \frac{p(A-B)}{1+B}-p(-1<B<A \leq 1 ; p \in \mathrm{N}) \text {. }
$$

If

$$
\left|\arg \left(-\frac{z\left(I_{n+p-1, \eta} f(z)\right)^{\prime}}{I_{n+p-1, \eta} g(z)}-\ell\right)\right|<\frac{\pi}{2} \delta,
$$

for some $g(z) \in \sum_{p, m}$ satisfying (4.6), then

$$
\left|\arg \left(-\frac{z\left(I_{n+p, \eta} f(z)\right)^{\prime}}{I_{n+p, \eta} g(z)}-\ell\right)\right|<\frac{\pi}{2} \tau,
$$

where $\tau(0<\tau \leq 1)$ is the solution of equation (4.1).

Taking $p=1$ in Corollary 7, we have the following corollary:

Corollary 8. Let $f(z) \in \sum, \quad 0<\delta \leq 1,0 \leq \ell<1$ and

$$
n \geq \frac{(A-B)}{1+B}-1 \quad(-1<B<A \leq 1) .
$$

If

$$
\left|\arg \left(-\frac{z\left(I_{n, \eta} f(z)\right)^{\prime}}{I_{n, \eta} g(z)}-\ell\right)\right|<\frac{\pi}{2} \delta,
$$

for some $g(z) \in \sum$ satisfying (4.7), then

$$
\left|\arg \left(-\frac{z\left(I_{n+1, \eta} f(z)\right)^{\prime}}{I_{n+1, \eta} g(z)}-\ell\right)\right|<\frac{\pi}{2} \tau,
$$

where $\tau(0<\tau \leq 1)$ is the solution of equation (4.1).

The proof of the next theorem is akin to that of Theorem 5 and so, we omit it.

Theorem 6. Let $f(z) \in \sum_{p, m}, \quad 0<\delta \leq 1, \ell>p$ and 


$$
\alpha_{1} \geq \frac{p(A-B)}{1+B}(-1<B<A \leq 1 ; p \in \mathrm{N})
$$

If

$$
\left|\arg \left(\frac{z\left(\mathbf{M}_{p, q, s}^{\lambda, m}\left(\alpha_{1}\right) f(z)\right)^{\prime}}{\mathbf{M}_{p, q, s}^{\lambda, m}\left(\alpha_{1}\right) g(z)}+\ell\right)\right|<\frac{\pi}{2} \delta,
$$

for some $g(z) \in \sum_{p, q, s}^{\lambda, m}\left(\alpha_{1} ; A, B\right)$, then

$$
\left|\arg \left(\frac{z\left(\mathbf{M}_{p, q, s}^{\lambda, m}\left(\alpha_{1}+1\right) f(z)\right)^{\prime}}{\mathbf{M}_{p, q, s}^{\lambda, m}\left(\alpha_{1}+1\right) g(z)}+\ell\right)\right|<\frac{\pi}{2} \tau,
$$

where $\tau(0<\tau \leq 1)$ is the solution of equation (4.1).

Theorem 7. Let $f(z) \in \sum_{p, m}, \quad 0<\delta \leq 1,0 \leq \ell<p$ and

$$
\mu \geq \frac{p(A-B)}{1+B}(-1<B<A \leq 1 ; p \in \mathrm{N}) .
$$

If

$$
\mid \arg \left(-\frac{z\left(\mathbf{M}_{p, q, s}^{\lambda, m}\left(\alpha_{1}\right) f(z)\right)^{\prime}}{\mathbf{M}_{p, q, s}^{\lambda, m}\left(\alpha_{1}\right) g(z)}-\ell\right)<\frac{\pi}{2} \delta,
$$

for some $g(z) \in \sum_{p, q, s}^{\lambda, m}\left(\alpha_{1} ; A, B\right)$, then

$$
\mid \arg \left(-\frac{z\left(\mathbf{M}_{p, q, s}^{\lambda, m}\left(\alpha_{1}\right) F_{\mu, p}(f)(z)\right)^{\prime}}{\mathbf{M}_{p, q, s}^{\lambda, m}\left(\alpha_{1}\right) F_{\mu, p}(g)(z)}-\ell\right)<\frac{\pi}{2} \tau,
$$

where $\tau(0<\tau \leq 1)$ is the solution of equation (4.1).

Proof. Let

$$
k(z)=\frac{1}{p-\ell}\left(-\frac{z\left(\mathbf{M}_{p, q, s}^{\lambda, m}\left(\alpha_{1}\right) F_{\mu, p}(f)(z)\right)^{\prime}}{\mathbf{M}_{p, q, s}^{\lambda, m}\left(\alpha_{1}\right) F_{\mu, p}(g)(z)}-\ell\right),
$$

where $k(z)$ is analytic with $k(0)=1$. Using (1.9), we have

$$
[-(p-\ell) k(z)-\ell] \mathbf{M}_{p, q, s}^{\lambda, m}\left(\alpha_{1}\right) F_{\mu, p}(g)(z)=\mu \mathbf{M}_{p, q, s}^{\lambda, m}\left(\alpha_{1}\right) f(z)-(\mu+p) \mathbf{M}_{p, q, s}^{\lambda, m}\left(\alpha_{1}\right) F_{\mu, p}(f)(z) . \text { (4.12) }
$$

Differentiating (4.12) with respect to $z$ and multiplying by $z$, we obtain

$$
-(p-\ell) z k^{\prime}(z) \mathbf{M}_{p, q, s}^{\lambda, m}\left(\alpha_{1}\right) F_{\mu, p}(g)(z)+[-(p-\ell) k(z)-\ell] z\left(\mathbf{M}_{p, q, s}^{\lambda, m}\left(\alpha_{1}\right) F_{\mu, p}(g)(z)\right)^{\prime}
$$




$$
=\mu z\left(\mathbf{M}_{p, q, s}^{\lambda, m}\left(\alpha_{1}\right) f(z)\right)^{\prime}-(\mu+p) z\left(\mathbf{M}_{p, q, s}^{\lambda, m}\left(\alpha_{1}\right) F_{\mu, p}(f)(z)\right)^{\prime} .
$$

Then, by using (4.11), (4.12) and (4.13), we have

$$
\frac{1}{p-\ell}\left(-\frac{z\left(\mathbf{M}_{p, q, s}^{\lambda, m}\left(\alpha_{1}\right) f(z)\right)^{\prime}}{\mathbf{M}_{p, q, s}^{\lambda, m}\left(\alpha_{1}\right) g(z)}-\ell\right)=k(z)+\frac{z k^{\prime}(z)}{-\rho(z)+\mu+p},
$$

where

$$
\rho(z)=-\frac{z\left(\mathbf{M}_{p, q, s}^{\lambda, m}\left(\alpha_{1}\right) F_{\mu, p}(g)(z)\right)^{\prime}}{\mathbf{M}_{p, q, s}^{\lambda, m}\left(\alpha_{1}\right) F_{\mu, p}(g)(z)} .
$$

The remaining part of the proof is similar to that of Theorem 3 and so we omit it.

The proof of the next theorem is akin to that of Theorem 7 and so, we omit it.

Theorem 8. Let $f(z) \in \sum_{p, m}, \quad 0<\delta \leq 1, \ell>p$ and

$$
\mu \geq \frac{p(A-B)}{1+B}(-1<B<A \leq 1 ; p \in \mathrm{N})
$$

If

$$
\left|\arg \left(\frac{z\left(\mathbf{M}_{p, q, s}^{\lambda, m}\left(\alpha_{1}\right) f(z)\right)^{\prime}}{\mathbf{M}_{p, q, s}^{\lambda, m}\left(\alpha_{1}\right) g(z)}+\ell\right)\right|<\frac{\pi}{2} \delta
$$

for some $g(z) \in \sum_{p, q, s}^{\lambda, m}\left(\alpha_{1} ; A, B\right)$, then

$$
\left|\arg \left(\frac{z\left(\mathbf{M}_{p, q, s}^{\lambda, m}\left(\alpha_{1}\right) F_{\mu, p}(f)(z)\right)^{\prime}}{\mathbf{M}_{p, q, s}^{\lambda, m}\left(\alpha_{1}\right) F_{\mu, p}(g)(z)}+\ell\right)\right|<\frac{\pi}{2} \tau
$$

where $\tau(0<\tau \leq 1)$ is the solution of equation (4.1).

Remark 1. Specializing the parameters $p, q, s, m$ and $\lambda$ in the above results, we obtain the results for the corresponding operators defined in the introduction.

Remark 2. (i) Putting $q=2, s=1, m=1-p, \quad \alpha_{1}=\beta_{1}=a(a>0, p \in \mathrm{N})$ and $\alpha_{2}=1$ in Theorems 3 and 4, respectively, we obtain the results obtained by Cho and Owa [11, Theorems 2.1 and 2.2, respectively] ; (ii) Putting $q=2, s=1, m=0, \quad \alpha_{1}=a, \alpha_{2}=p=1$ and $\beta_{1}=a \quad(a>0) \quad$ in Theorems 3 and 4, respectively, we obtain the results obtained by Cho [8, Theorems 2.1 and 2.2, respectively] ; (iii) Putting $q=2, s=1, m=1-p, \quad \alpha_{1}=a, \alpha_{2}=1$ and $\beta_{1}=c \quad\left(a, c \in \mathrm{R} \backslash \mathrm{Z}_{0}^{-}, p \in \mathrm{N}\right)$ in the above results, we obtain the results obtained by Aouf et al. [5] ; 
(iv) Putting $q=2, s=1, m=1-p, \alpha_{1}=c, \alpha_{2}=p+\lambda$ and $\beta_{1}=a\left(a \in \mathrm{R}, c \in \mathrm{R} \backslash \mathrm{Z}_{0}^{-}, \lambda>-p, p \in \mathrm{N}\right)$ in Theorem 4, we obtain the results obtained by Lashin [14, Theorem 2.2] .

\section{References}

[1] R. Aghalary, Some properties of a certain family of meromorphically univalent functions defined by integral operator, Kyungpook Math. J., 48 (2008), 379-385.

[2] M. K. Aouf, New criteria for multivalent meromorphic starlike functions of order alpha, Proc. Japan Acad., Ser. A, Math. Sci., 69 (1993), 66-70.

[3] M. K. Aouf, A new criterion for meromorphically p-valent convex functions of order alpha, Math. Sci. Research Hot-Line, 1 (1997), no. 8, 7-12.

[4] M.K. Aouf, Certain subclasses of meromorphically multivalent functions associated with generalized hypergeometric function, Comput. Math. Appl., 55 (2008), 494-509.

[5] M.K. Aouf, A. Shamandy, A.O. Mostafa and F.Z. El-Emam, Argument estimates of certain meromorphically $p$-valent functions associated with a family of linear operator, Math. Slovaca, 61 (2012), no. 6, 907-920.

[6] M. K. Aouf and N.- E. Xu, Some inclusion relationships and integral-preserving properties of certain subclasses of p-valent meromorphic functions, Comput. Math. Appl., 61 (2011), 642-650.

[7] T. Bulboaca, Differential Subordinations and Superordinations, Recent Results, House of Scientific Book Publ., Cluj-Napoca, 2005.

[8] N. E. Cho, Argument estimates of certain meromorphic functions, Comm. Korean Math. Soc., 15 (2000), no. 2, 263-274.

[9] N. E. Cho and I. H. Kim, Inclusion properties of certain classes of meromorphic functions associated with the generalized hypergeometric function, Appl. Math. Comput., 187 (2007), 115-121.

[10] N. E. Cho and K. I. Noor, Inclusion properties for certain classes of meromorphic functions associated with the Choi-Saigo-Srivastava operator, J. Math. Anal. Appl., 320, (2006), 779-786.

[11] N. E. Cho and S. Owa, Argument estimates of meromorphically multivalent functions, J. Inequal. Appl., 5 (2000), 419-432.

[12] P. Eenigenberg, S. S. Miller, P. T. Mocanu and M. O. Reade, On a Briot--Bouquet differential subordination, General Inequal., 3 (1983), 339-348.

[13] V. Kumar and S. L. Shukla, Certain integrals for classes of p-valent meromorphic functions, Bull. Austral. Math. Soc., 25 (1982), 85-97.

[14] A. Y. Lashin, Argument estimates of certain meromorphically p-valent functions, Soochow J. Math., 33 (2007), no. 4, 803-812.

[15] J.-L. Liu, A linear operator and its applications on meromorphic p-valent functions, Bull. Inst. Math. Acad. Sinica, 31 (2002), no. 1, 23-32. 
[16] J.-L. Liu and H. M. Srivastava, Classes of meromorphically multivalent functions associated with generalized hypergeometric function, Math. Comput. Modelling, 39 (2004), 21-34.

[17] A. O. Mostafa, Applications of differential subordination to certain subclasses of $p$-valent meromophic functions involving certain operator, Math. Comput. Modelling, 54 (2011), 1486-1498.

[18] S. S. Miller and P. T. Mocanu, Differential subordinations and univalent functions, Michigan Math. J., 28 (1981), 157-171.

[19] M. Nunokawa, On the order of strongly starlikeness of strongly convex functions, Proc. Japan Acad., Ser. A, Math. Sci., 69 (1993), 234-237.

[20] J. Patel and A. K. Patil, On certain subclasses of meromorphically multivalent functions associated with the generalized hypergeometric function, J. Inequal. Pure Appli. Math., 10 (2009), no. 1, Art 13, 1-33.

[21] H. Silverman and E. M. Silvia, Subclasses of starlike functions subordinate to convex functions, Canad. J. Math., 37 (1985), 48-61.

[22] H. M. Srivastava and P.W. Karlsson, Multiple Gaussian Hypergeometric Series, Halsted Press, Ellis Horwood Limited, Chichester, John Wiley and Sons, New York, Chichester, Brisbane, Toronto, 1985.

[23] D. -G. Yang, Certain convolution operators for meromorphic functions, South. Asian Bull. Math., 25 (2001), 175-186.

[24] S.-M. Yuan, Z. -M. Liu and H. M. Srivastava, Some inclusion relationships and integral-preserving properties of certain subclasses of meromorphic functions associated with a family of integral operators, J. Math. Anal. Appl., 337 (2008), 505-515. 